\title{
Galectin-3 Reflects the Echocardiographic Grades of Left Ventricular Diastolic Dysfunction
}

\begin{abstract}
Uzair Ansari, M.D. ${ }^{1 *}$, Michael Behnes, M.D. ${ }^{1 *}$, Julia Hoffmann, M.S. ${ }^{1}$, Michele Natale, M.S. ${ }^{1}$, Christian Fastner, M.D. ${ }^{1}$, Ibrahim El-Battrawy, M.D. ${ }^{1}$, Jonas Rusnak, M.D. ${ }^{1}$, Seung-Hyun Kim, M.D. ${ }^{1}$, Siegfried Lang, Ph.D. ${ }^{1}$, Ursula Hoffmann, M.D. ${ }^{1}$, Thomas Bertsch, M.D. ${ }^{2}$, Martin Borggrefe, M.D. ${ }^{1}$, and Ibrahim Akin, M.D. ${ }^{1}$

First Department of Medicine ${ }^{1}$, University Medical Center Mannheim, University of Heidelberg, Mannheim, Germany: DZHK (German Center for Cardiovascular Research) partner site Mannheim; Institute of Clinical Chemistry, Laboratory Medicine and Transfusion Medicine², General Hospital Nuremberg, Paracelsus Medical University, Nuremberg, Germany
\end{abstract}

Background: The level of Galectin-3 (Gal-3) protein purportedly reflects an ongoing cardiac fibrotic process and has been associated with ventricular remodeling, which is instrumental in the development of heart failure with preserved ejection fraction (HFpEF) syndrome. The aim of this study was to investigate the potential use of Gal-3 in improved characterization of the grades of diastolic dysfunction as defined by echocardiography.

Methods: Seventy HFpEF patients undergoing routine echocardiography were prospectively enrolled in the present monocentric study. Blood samples for measurements of Gal-3 and amino-terminal pro-brain natriuretic peptide (NT-proBNP) were collected within 24 hours pre- or post-echocardiographic examination. The classification of patients into subgroups based on diastolic dysfunction grade permitted detailed statistical analyses of the derived data.

Results: The Gal-3 serum levels of all patients corresponded to echocardiographic indices, suggesting HFpEF (E/A, $P=0.03$ and E/E', $P=0.02$ ). Gal-3 was also associated with progressive diastolic dysfunction, and increased levels corresponded to the course of disease $(P=0.012)$. Detailed analyses of ROC curves suggested that Gal-3 levels could discriminate patients with grade III diastolic dysfunction (area under the curve $[A \cup C]=0.770$, $P=0.005$ ).

Conclusions: Gal-3 demonstrates remarkable effectiveness in the diagnosis of patients suffering from severe grade diastolic dysfunction. Increasing levels of Gal-3 possibly reflect the progressive course of $\mathrm{HFpEF}$, as classified by the echocardiographic grades of diastolic dysfunction.

Key Words: Galectin-3, Preserved ejection fraction, NT-proBNP, Diastolic dysfunction

\author{
Received: July 26, 2017 \\ Revision received: October 19, 2017 \\ Accepted: January 15, 2018
}

\begin{abstract}
Corresponding author: Uzair Ansari University Medical Center Mannheim, First Department of Medicine, TheodorKutzer-Ufer 1-3, 68167 Mannheim, Germany

Tel: +49-621-383-5229

Fax: +49-621-383-2012

E-mail: uzair.ansari@umm.de
\end{abstract}

*These authors contributed equally to this study.

\begin{abstract}
(๔) Korean Society for Laboratory Medicine
This is an Open Access article distributed under the terms of the Creative Commons Attribution Non-Commercial License (http://creativecommons.org/licenses/by-nc/4.0) which permits unrestricted non-commercial use, distribution, and reproduction in any medium, provided the original work is properly cited.
\end{abstract}

\section{INTRODUCTION}

The prevalence of heart failure with preserved ejection fraction (HFpEF) has increased significantly over the past decade [1, 2]. Hypothesis suggesting that HFpEF is an independent syndrome typically characterized by the presence of diastolic dysfunction has not ameliorated the ominous prognosis of this presentation.
The reported mortality rates are as high as $\sim 65 \%$ at five years post initial hospitalization [3]. It is estimated that $>50 \%$ of all patients presenting with signs and symptoms of heart failure could have a preserved ejection fraction (EF) [4]. Myocardial stiffening from hypertrophy and fibrosis are central to the traditional paradigm of HFpEF; however, the roles of abnormal calcium handling and venous turgor have also been exposed in re- 
cent studies [5-7]. The pathophysiological factors implicated in the development of this syndrome include impaired relaxation (attributed to low-grade inflammation, extracellular matrix accumulation, and fibrosis), increased left ventricular stiffness and reduced compliance, atrial dysfunction, chronotropic incompetence, pre- and post-capillary pulmonary hypertension, and vascular stiffening $[4,8]$.

The use of serum biomarkers has helped provide vital information regarding the pathogenesis of HFpEF and is a proven clinical tool for the identification of at-risk patients, syndrome diagnosis, risk stratification, as well as therapy monitoring [3]. Galectin-3 (Gal-3), a similar serum biomarker, is a soluble $\beta$-galactoside-binding protein secreted by activated macrophages and is a key component in chronic inflammation facilitating fibrogenesis and organ scarring [9]. The hypothesis that Gal-3 influences the onset of heart failure has been corroborated by infusing Gal-3 into the pericardial sac of wild-type rats, which triggered extensive myocardial fibrosis. It has also been suggested that Gal-3 induces cardiac fibrosis via activation of cyclin D1, thus enabling a macrophage derived mediator to affect the myocardium [10]. Additionally, Gal-3 complements other similar heart failure biomarkers, such as aminoterminal pro-brain natriuretic peptide (NT-proBNP) and troponin T and troponin I (TnT/Tnl), by providing an upstream signal of the myocardial fibrotic state, ventricular adverse remodeling, and cardiomyopathy progression [11].

The predominant use of echocardiography to assess impaired myocardial diastolic function is naturally influenced by patient compliance, adiposity, and pulmonary diseases such as emphysema. The use of biomarkers to determine grades of diastolic dysfunction is not subject to these limitations. Recent studies have attempted to delineate the potential relationship between Gal-3 levels and transthoracic echocardiographic indices such as left ventricular EF (LVEF) and right ventricular (RV) systolic pressure [12]. Expanding on this idea, our study attempted to explore the hitherto poorly investigated hypothesis that quantitative levels of Gal-3 could also reflect other echocardiographic indices defined in the evolving stages of HFpEF.

\section{METHODS}

\section{Study design and population characteristics}

The Cardiovascular Imaging and Biomarker Analyses (CIBER) study (clinicaltrails.gov identifier: NCT 03074253) is a clinically prospective, controlled, and mono-centric study conducted at the University Medical Center Mannheim, Germany. The research adhered to the principles outlined in the Declaration of Helsinki and was approved by a regional ethics committee (the medical ethics commission II of the Faculty of Medicine in Mannheim, University of Heidelberg, Germany). Written informed consent was obtained from all patients.

The present study incorporated a population subset derived from a patient cohort who underwent routine echocardiography at the University Medical Centre Mannheim, Germany between 2014 and 2016. A total of 70 patients diagnosed with HFpEF were included consecutively in this mono-centric, prospective study with an all-comers design. As this was a non-interventional, observational study, diagnostic procedures and treatment plans were not modified.

The relevant clinical data of each patient were ascertained and compiled in a database at index presentation, with significant aspects of their medical history, laboratory work-up, and medi$\mathrm{cal} /$ surgical therapy efficiently earmarked for further reference. Patients under the age of 18 years or those with LVEF $<50 \%$, tricuspid annular plane systolic excursion (TAPSE) $<17 \mathrm{~mm}$, and/or valvular heart disease were excluded. Blood samples, collected from all patients, were preserved and processed throughout the study. All patients were contacted at the scheduled 6and 12-month follow-up period for standardized telephonic interviews to ascertain the incidence of re-hospitalization due to heart failure as well as all-cause and cardiovascular mortality.

\section{Echocardiography}

A detailed transthoracic echocardiographic examination was performed for all patients included, and standard techniques were implemented to acquire every reproducible image [13, 14]. The results were analyzed and interpreted by observers with no knowledge of the patients' clinical and biomarker data. The assessed structural indices included LVEF (using Simpsons biplane method), left ventricular (LV) end-systolic and end-diastolic volume, LV wall and septal thickness, atrial and ventricular size and volume, TAPSE, markers of early and late trans-mitral diastolic velocities ( $E$ and $A$ ), deceleration time, and early and late diastolic tissue velocities at the lateral mitral annulus ( $E^{\prime}$ ) [12]. Patients were classified according to the grade of diastolic dysfunction, and grading guidelines were based on the American Society of Echocardiography and European Association of Cardiovascular Imaging (ASE/EACVI) Guidelines recommended by Nagueh [13]. A flow diagram depicting our diagnostic approach for the inclusion of patients is shown in Supplemental Data Fig. S1.

The echocardiographic inclusion criteria specified that pa- 
tients have a preserved LV and right ventricular (RV) function. Consequently, patients with an LVEF $<50 \%$ and TAPSE $<17$ $\mathrm{mm}$ were excluded. Additionally, patients with moderate and severe heart valve disorders, classified as either stenosis or regurgitation, were excluded.

\section{Laboratory analysis}

Blood samples were collected from all patients (at rest) at a single assessment time point upon study inclusion by venepuncture with serum monovettes and centrifuged at 2,500 $\mathrm{g}$ at $20^{\circ} \mathrm{C}$ for 10 minutes. The aliquoted samples were cooled down in liquid nitrogen before being stored at $-80^{\circ} \mathrm{C}$ for further analysis. After thawing, the samples were mixed gently by inverting and centrifuged at 2,500 $\mathrm{g}$ for 10 minutes at $20^{\circ} \mathrm{C}$.

Gal-3 levels were assessed using the Gal-3 assay on an Architect i1000 analyzer (Abbott, Wiesbaden, Germany). The limit of blank for this assay was $0.8 \mathrm{ng} / \mathrm{mL}$, as specified in the user instructions (Galectin-3, Architect System, () 2012, 2013 Abbott Laboratories). Serum creatinine concentrations were measured using the Creatinine Jaffe Gen.2 assay on a Cobas c 702 analyzer (Roche Diagnostics, Mannheim, Germany), and the glomerular filtration rate (eGFR) was estimated using the Modification of Diet in Renal disease (MDRD) formula (Instructions for use, Cobas c 702 analyzer). The serum level of NT-proBNP, used as a reference biomarker, was measured using a proBNP II STAT assay on a Cobas e 602 analyzer (Roche Diagnostics). The limit of detection (LoD) for this assay was $5 \mathrm{pg} / \mathrm{mL}$ (proBNP II STAT, Cobas $^{\circledR}$, ( 2014 , Roche Diagnostics).

\section{Statistical analysis}

The Student t-test was applied for data with a normal distribution, and the Kruskal-Wallis method was used as a non-parametric test. Abnormally distributed scaled variables with significant deviations from the Gaussian distribution were compared using the Kolmogorov-Smirnov test. Spearman's rank correlation for non-parametric data was used to test the association of Gal-3 serum levels with cardiac indices and other parameters, as defined by transthoracic echocardiography. The data are presented as the mean with a confidence interval $(\mathrm{Cl})$ or median with interquartile ranges (IQRs; 25th to 75th percentiles), depending on the distribution. $P<0.05$ was considered statistically significant.

The effectiveness of Gal-3 in grading the various stages of HFpEF was evaluated using the Hanley and McNeil method [17], with the reference biomarker, NT-proBNP, plotted simultaneously for comparison. The data were further log-transformed
Table 1. Baseline characteristics of study patients

\begin{tabular}{lc}
\hline Characteristics & HF-PEF (N=70) \\
\hline Age, mean (range; 95\% Cl) & $65(22-97 ; 84)$ \\
Gender, N (\%) & \\
Male & $36(51)$ \\
Female & $34(49)$ \\
Cardiovascular risk factors, N (\%) & \\
Arterial hypertension & $56(80)$ \\
Hypercholesterinemia & $26(37)$ \\
Cardiac family history & $11(16)$ \\
Smoking status & $23(33)$ \\
Diabetes mellitus & $17(24)$ \\
Adipositas & $16(23)$ \\
Laboratory parameters, median (IQR) & \\
Creatinine (mg/dL) & $0.93(0.77-1.15)$ \\
eGFR (mL/min/1.73 m²) & 70.47 \\
Medical history, N (\%) & \\
Chronic heart failure & $50(71)$ \\
NYHA I & $22(31)$ \\
NYHA II & $10(14)$ \\
NYHA III & $17(24)$ \\
NYHA IV & $1(1)$ \\
Atrial fibrillation & $27(38)$ \\
Paroxysmal & $15(21)$ \\
Persistent & $9(13)$ \\
Permanent & $3(4)$ \\
Coronary artery disease & $35(50)$ \\
1 vessel disease & $4(6)$ \\
Myosesel disease disease & $12(17)$ \\
Chronic kidney disease & $19(27)$ \\
COPD & $5(7)$ \\
Asthma & $12(17)$ \\
Cancer & $8(11)$ \\
\hline & $2(3)$ \\
Ab & $18(26)$ \\
\hline
\end{tabular}

Abbreviations: HFpEF, heart failure with preserved ejection fraction; COPD, chronic obstructive pulmonary disease; NYHA, New York Heart Association; GFR, glomerular filtration rate.

for analysis. Potential confounding factors were defined using multivariable linear or logistic regression analyses with backward elimination, and clinical parameters or biomarkers were adjusted depending on the outcome variable (binary or numeric).

Statistical analyses were performed using SPSS Statistics (IBM, Armonk, NY, USA) and GraphPad Prism (GraphPad Software, 
Table 2. Distribution of echocardiographic indices according to HFpEF sub-groups

\begin{tabular}{|c|c|c|c|c|c|c|}
\hline & $\begin{array}{l}\text { All Patients } \\
(\mathrm{N}=70)\end{array}$ & $\begin{array}{l}\text { Good Diastolic Function } \\
\qquad(\mathrm{N}=14)\end{array}$ & $\begin{array}{l}\text { Diastolic Dysfunction I } \\
\quad(\mathrm{N}=15)\end{array}$ & $\begin{array}{l}\text { Diastolic Dysfunction II } \\
(\mathrm{N}=30)\end{array}$ & $\begin{array}{l}\text { Diastolic Dysfunction III } \\
\qquad(\mathrm{N}=11)\end{array}$ & $P$ \\
\hline LVEF (\%) & $59.00(56.00-65.00)$ & $61.00(56.00-65.00)$ & $58.00(57.00-62.00)$ & $62.00(56.00-67.00)$ & $56.00(54.00-58.00)$ & 0.109 \\
\hline LVEDD (mm) & $45.00(43.00-50.00)$ & $45.00(42.00-46.00)$ & $44.00(43.00-48.00)$ & $46.00(40.00-50.00)$ & $50.00(45.00-58.00)$ & 0.112 \\
\hline LVESD (mm) & $30.00(27.00-34.00)$ & $30.00(26.00-32.00)$ & $29.00(27.00-35.00)$ & $28.00(27.00-32.00)$ & $31.00(28.00-37.00)$ & 0.39 \\
\hline LVPW (mm) & $11.00(9.00-12.00)$ & $10.00(8.00-11.00)$ & $10.00(9.00-12.00)$ & $11.00(10.00-12.00)$ & $12.00(11.00-14.00)$ & 0.045 \\
\hline $\operatorname{LVIS}(\mathrm{mm})$ & $12.00(10.00-13.00)$ & $11.00(9.00-12.00)$ & $11.00(10.00-12.00)$ & $12.00(10.00-13.00)$ & $13.00(12.00-13.00)$ & 0.065 \\
\hline $\mathrm{RA}(\mathrm{mm})$ & $36.00(33.00-40.00)$ & $35.00(31.00-40.00)$ & $37.00(35.00-38.00)$ & $35.00(32.00-40.00)$ & $36.00(34.00-41.00)$ & 0.911 \\
\hline $\mathrm{RA}\left(\mathrm{cm}^{3}\right)$ & $14.00(12.00-17.00)$ & $13.00(11.00-16.00)$ & $15.00(13.00-16.00)$ & $14.00(12.00-15.00)$ & $17.00(14.00-18.00)$ & 0.172 \\
\hline $\mathrm{LA}(\mathrm{mm})$ & $39.00(35.00-43.00)$ & $35.00(34.00-38.00)$ & $40.00(36.00-45.00)$ & $39.00(35.00-41.00)$ & $43.00(40.00-47.00)$ & 0.044 \\
\hline $\mathrm{LA}\left(\mathrm{cm}^{2}\right)$ & $17.00(15.00-19.00)$ & $15.00(11.00-17.00)$ & $17.00(16.00-18.00)$ & $17.00(15.00-20.00)$ & $24.00(20.00-27.00)$ & 0.002 \\
\hline $\mathrm{RV}$-area $\left(\mathrm{cm}^{2}\right)$ & $18.00(16.00-21.00)$ & $18.00(14.00-21.00)$ & $18.00(17.00-20.00)$ & $18.00(16.00-21.00)$ & $19.00(15.00-22.00)$ & 0.926 \\
\hline RV-volume (mL) & $37.00(29.00-49.00)$ & $34.00(25.00-43.00)$ & $40.00(31.00-44.00)$ & $37.00(29.00-52.00)$ & $38.00(26.00-53.00)$ & 0.634 \\
\hline LV-area $\left(\mathrm{cm}^{2}\right)$ & $35.00(31.00-40.00)$ & $33.00(28.00-35.00)$ & $39.00(31.00-44.00)$ & $34.00(31.00-39.00)$ & $37.00(33.00-42.00)$ & 0.099 \\
\hline LV-volume (mL) & $116.00(92.00-151.00)$ & $114.00(89.00-130.00)$ & $149.00(99.00-170.00)$ & $111.00(90.00-137.00)$ & $128.00(92.00-176.00)$ & 0.218 \\
\hline Aorta (mm) & $29.00(27.00-33.00)$ & $28.00(26.00-31.00)$ & $28.00(26.00-38.00)$ & $30.00(27.00-33.00)$ & $31.00(29.00-32.00)$ & 0.466 \\
\hline AortaP $P_{\text {mean }}(\mathrm{mmHg})$ & $8.50(7.00-11.00)$ & $8.00(8.00-8.00)$ & $9.00(9.00-9.00)$ & $9.80(5.50-14.00)$ & $9.00(7.00-11.00)$ & 0.986 \\
\hline AortaP ${ }_{\text {max }}(\mathrm{mmHg})$ & $7.00(6.00-10.00)$ & $6.00(5.00-7.00)$ & $9.00(6.00-10.00)$ & $8.00(6.00-10.00)$ & $7.00(6.00-13.00)$ & 0.236 \\
\hline TAPSE (mm) & $22.00(20.00-25.00)$ & $22.00(21.00-25.00)$ & $21.00(19.00-23.00)$ & $23.00(21.00-26.00)$ & $20.00(19.00-18.00)$ & 0.41 \\
\hline $\mathrm{E} / \mathrm{A}(\mathrm{cm} / \mathrm{s})$ & $0.90(0.70-1,20)$ & $1.20(1.10-1.50)$ & $0.80(0.60-0.90)$ & $0.80(0.70-1.10)$ & $0.90(0.80-1.30)$ & 0.0001 \\
\hline E'med (m/s) & $0.06(0.05-0.08)$ & $0.11(0.09-0.13)$ & $0.07(0.05-0.08)$ & $0.06(0.05-0.08)$ & $0.04(0.04-0.06)$ & 0.002 \\
\hline E'lat (m/s) & $0.07(0.06-0.09)$ & $0.10(0.08-0.13)$ & $0.09(0.07-0.11)$ & $0.08(0.06-0.09)$ & $0.05(0.05-0.06)$ & 0.009 \\
\hline$E / E^{\prime}$ & $10.00(8.00-13.00)$ & $6.00(4.00-7.00)$ & $7.00(6.00-8.00)$ & $11.00(10.00-12.00)$ & $19.00(16.00-26.00)$ & 0.0001 \\
\hline DT (ms) & $237.00(181.00-296.00)$ & $214.00(164.00-237.00)$ & $243.00(187.00-299.00)$ & $240.00(205.00-296.00)$ & $236.00(192.00-373.00)$ & 0.119 \\
\hline
\end{tabular}

Data are presented as medians with interquartile ranges (IQRs); bold type indicates statistical significance $(P<0.05)$.

Abbreviations: LVEF, left ventricular ejection fraction; LVEDD, left ventricular end diastolic diameter; LVESD, left ventricular end systolic diameter; LVEDV, LV end-diastolic volume; LVESV, LV end-systolic volume; LVPW, LV posterior wall; IVSD, interventricular septal diameter; RA, right atrium; LA, left atrium; RV, right ventricle; LV, left ventricle; TAPSE, tricuspid annular plane systolic excursion; E/A, ratio of the early $(E)$ to late $(A)$ ventricular filling velocities; E/E', ratio of mitral inflow (E) velocity to tissue Doppler (E'); DT, deceleration time; IVRT, isovolumetric relaxation time.

Inc., La Jolla, CA, USA). All patient data resulting from these analyses were subsequently interpreted and classified into the three sub-groups defined by HFpEF grade (I, II, III). The details of this classification and the corroborating echocardiographic measurements are provided in Tables 1 and 2 .

\section{RESULTS}

\section{Study population}

The baseline clinical characteristics of the 70 patients are described in Table 1. A detailed analysis of the data revealed that the mean age of the patients was 65 years (range 22-97 years) with an equal gender distribution (male $51 \%, \mathrm{~N}=36$; female $49 \%, N=34$ ). Arterial hypertension was identified as a predominant risk factor in this group, with $80 \%(\mathrm{~N}=56)$ of the patients diagnosed as having this disease. Patients suffering from heart failure-related symptoms represented $71 \%(\mathrm{~N}=50)$ of the study population, and their sub-classification into New York Heart Association (NYHA) sub-groups yielded an almost even distribution across classes I, II, and III. Coronary artery disease was identified as a pre-existing condition in at least $50 \%(N=35)$ of the patients, while atrial fibrillation was documented in $38 \%(\mathrm{~N}=27)$. Patients suffering from diabetes mellitus $(24 \%, N=17)$ or chronic kidney disease $(17 \%, \mathrm{~N}=12)$ were also represented. The median creatinine value was estimated at $0.93 \mathrm{mg} / \mathrm{dL}$ (range $0.77-$ $1.15 \mathrm{mg} / \mathrm{dL}$ ) and the median eGFR according to MDRD formula was $70.47 \mathrm{~mL} / \mathrm{min} / 1.73 \mathrm{~m}^{2}$.

\section{Echocardiographic characteristics}

The distribution of echocardiographic indices according to HF- 

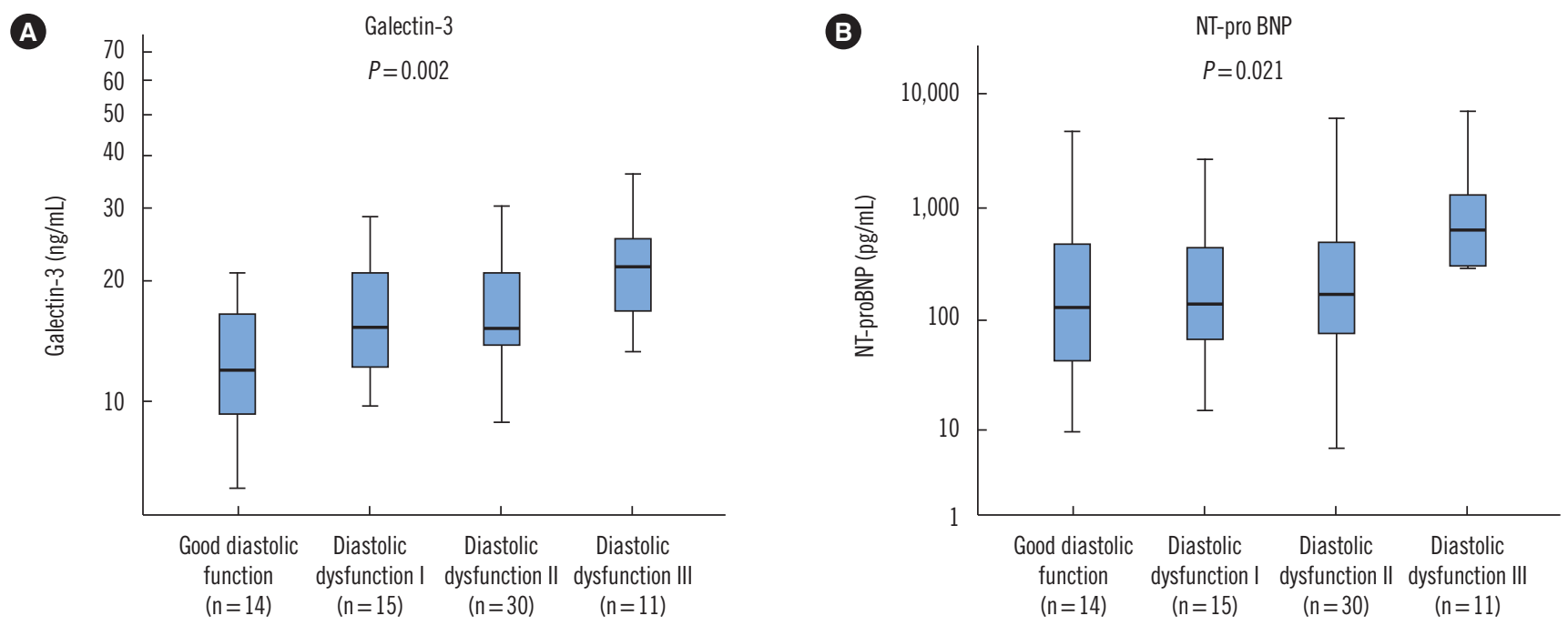

Fig. 1. Box plots showing increased levels of Galectin-3 (A) and increased levels of NT-proBNP (B) in patients with different grades of diastolic dysfunction. Significantly highest levels of Galectin-3 and NT-proBNP were noted in Grade III diastolic dysfunction, indicating a progressive increase in their levels along the course of the disease. Abbreviation: NT-proBNP, amino-terminal pro-brain natriuretic peptide.

pEF sub-group is outlined in Table 2. Patients expressed either good diastolic function $(\mathrm{N}=14)$ or grade I $(\mathrm{N}=15)$, grade II $(\mathrm{N}=30)$, or grade III $(\mathrm{N}=11)$ diastolic dysfunction. LVEF values were $>50 \%$ and TAPSE was $>17 \mathrm{mmHg}$. Detailed analysis of these data revealed that LVEF values ranged between $54 \%$ and $67 \%$. Indices considered statistically significant included LV posterior wall thickness $(P=0.045)$, left atrial dimensions $(P=0.044)$, left atrial volume $(P=0.002)$, E/A ratio $(P=0.0001)$, E' lateral (E' lat) $(P=$ $0.009)$, and E/E' ratio $(P=0.0001)$.

\section{Characteristics of Gal-3}

Gal-3 mirrored progressive diastolic dysfunction and increased levels corresponded with the course of disease $(P=0.012)$. The distribution of Gal-3 and NT-proBNP levels is graphically presented in Fig. 1.

\section{Correlation of Gal-3 with baseline characteristics and echocardiographic indices}

Univariate linear regression analysis demonstrating the correlation of Gal-3 with the baseline characteristics, as well as echocardiographic indices is detailed in Table 3. The Gal-3 levels of all patients corresponded with age $(P=0.0001)$, as well as with echocardiographic indices, suggesting HFpEF (E/A, $P=0.03$ and $E / E ', P=0.02$ ). Additionally, there was a significant correlation between Gal-3 levels and indices measuring left atrial and LV dimensions in the case of grade III diastolic dysfunction ( $P$ values ranging from 0.006 to 0.01 ). However, this relationship was blurred in patients with normal or lower grades of diastolic dysfunction. Gal-3 levels also demonstrated a strong association with serum creatinine levels across all HFpEF sub-groups ( $P$ values ranging from 0.01 to 0.0001 ).

Interestingly, although a definitive relationship with NT-proBNP was observed in all patients ( $P=0.0001)$, this was statistically nonsignificant across the various HFpEF sub-groups defined by varying degrees of diastolic dysfunction.

\section{Gal-3 level discriminates patients with Grade III diastolic dysfunction}

Detailed analyses ROC curves suggested that Gal-3 level discriminates patients with grade III diastolic dysfunction (area under the curve $[A \cup C]=0.770, P=0.005$; Fig. 2). NT-proBNP also revealed such characteristics, but with a numerically greater AUC (AUC $=0.798, P=0.002$ ). This revealed no significant difference between the two AUCs. Additionally, the combination of Gal-3 and NT-proBNP exhibited a similar discrimination for this sub-group of HFpEF patients ( $A \cup C=0.798, P=0.002$ ).

\section{Multivariate logistic regression models}

Data were log-transformed for this analysis. Log-transformed Gal-3 and NT-proBNP levels were initially adjusted to multivariate logistic regression models with each other and then subsequently for age, gender, and serum creatinine (Table 4). Patients with Gal-3 levels $\geq 17.0 \mathrm{ng} / \mathrm{mL}$ were six times more likely to suffer from grade III diastolic dysfunction (adjusted odds ratio [OR]= 
Table 3. Univariate correlations between Galectin-3 and patient characteristics, biomarkers, and echocardiographic parameters according to HF-pEF sub-groups

\begin{tabular}{|c|c|c|c|c|c|c|c|c|c|c|}
\hline & \multicolumn{2}{|c|}{$\begin{array}{l}\text { All patients } \\
(\mathrm{N}=70)\end{array}$} & \multicolumn{2}{|c|}{$\begin{array}{l}\text { Normal diastolic function } \\
\qquad(\mathrm{N}=14)\end{array}$} & \multicolumn{2}{|c|}{$\begin{array}{l}\text { Diastolic dysfunction } \\
\text { grade I }(N=15)\end{array}$} & \multicolumn{2}{|c|}{$\begin{array}{l}\text { Diastolic dysfunction } \\
\text { grade II }(N=30)\end{array}$} & \multicolumn{2}{|c|}{$\begin{array}{l}\text { Diastolic dysfunction } \\
\text { grade III }(\mathrm{N}=11)\end{array}$} \\
\hline & $r$ & $P$ & $r$ & $P$ & $r$ & $P$ & $r$ & $P$ & $r$ & $P$ \\
\hline Age & 0.43 & $0.0001^{*}$ & 0.43 & 0.13 & 0.27 & 0.34 & 0.21 & 0.27 & -0.26 & 0.45 \\
\hline Creatinine & 0.62 & $0.0001^{*}$ & 0.66 & $0.01^{*}$ & 0.41 & 0.13 & 0.45 & $0.01^{*}$ & 0.09 & $0.0001^{*}$ \\
\hline NT-proBNP & 0.45 & $0.0001^{*}$ & -0.09 & 0.77 & 0.44 & 0.09 & 0.47 & $0.01^{*}$ & 0.22 & 0.52 \\
\hline LVEF & -0.24 & 0.042 & 0.18 & 0.55 & -0.50 & 0.06 & -0.11 & 0.57 & -0.33 & 0.33 \\
\hline LVEDD & 0.20 & 0.093 & 0.16 & 0.58 & 0.33 & 0.24 & 0.09 & 0.64 & 0.04 & 0.92 \\
\hline LVESD & 0.19 & 0.11 & 0.26 & 0.37 & 0.04 & 0.89 & 0.26 & 0.17 & -0.11 & 0.76 \\
\hline LVPW & 0.38 & $0.001^{*}$ & 0.18 & 0.55 & 0.16 & 0.57 & 0.32 & $0.08^{*}$ & 0.73 & $0.01^{*}$ \\
\hline LVIS & 0.46 & $0.0001^{*}$ & 0.39 & 0.17 & 0.17 & 0.54 & 0.43 & $0.017^{*}$ & 0.83 & $0.002^{*}$ \\
\hline RA & 0.10 & 0.418 & 0.26 & 0.36 & 0.14 & 0.63 & 0.01 & 0.95 & 0.28 & 0.41 \\
\hline LA & 0.37 & $0.001^{*}$ & 0.57 & $0.032^{*}$ & 0.17 & 0.55 & 0.14 & 0.46 & 0.78 & $0.005^{*}$ \\
\hline LA-area & 0.45 & $0.0001^{*}$ & 0.34 & 0.23 & -0.12 & 0.69 & 0.26 & 0.20 & 0.86 & $0.006^{*}$ \\
\hline RV & 0.03 & 0.83 & 0.26 & 0.36 & -0.07 & 0.80 & -0.09 & 0.64 & 0.16 & 0.64 \\
\hline RV-Volume & 0.10 & 0.41 & 0.46 & 0.098 & -0.08 & 0.79 & -0.05 & 0.79 & 0.14 & 0.69 \\
\hline LV & 0.21 & 0.09 & 0.33 & 0.27 & -0.21 & 0.49 & 0.03 & 0.89 & 0.55 & 0.13 \\
\hline LV-Volume & 0.08 & 0.49 & 0.39 & 0.166 & -0.27 & 0.33 & -0.09 & 0.64 & 0.14 & 0.69 \\
\hline Aorta & 0.12 & 0.30 & 0.10 & 0.768 & -0.08 & 0.77 & -0.06 & 0.74 & 0.06 & 0.85 \\
\hline$E / A$ & -0.26 & $0.03^{*}$ & -0.03 & 0.94 & -0.19 & 0.50 & -0.26 & 0.17 & -0.08 & 0.83 \\
\hline E/E' & 0.29 & $0.02^{*}$ & -0.48 & 0.23 & 0.12 & 0.67 & -0.36 & $0.049 *$ & -0.009 & 0.98 \\
\hline Dec. Time & 0.67 & 0.58 & 0.13 & 0.65 & -0.37 & 0.20 & 0.13 & 0.52 & -0.20 & 0.61 \\
\hline
\end{tabular}

*Statistical significance $(P<0.05)$.

Abbreviations: LVEF, left ventricular ejection fraction; LVEDD, left ventricular end diastolic diameter; LVESD, left ventricular end systolic diameter; LVPW, left ventricular posterior wall; LVIS, left ventricular interventricular septum; RA, right atrium; LA, left atrium; E/A, markers of early and late trans-mitral diastolic velocities ( $E$ and $A$ ), early and late diastolic tissue velocities at the lateral mitral annulus (E'); E/E', ratio of mitral inflow (E) velocity to tissue Doppler (e'); Hf$\mathrm{pEF}$, Heart failure with preserved ejection fraction.

Table 4. Multivariable logistic regression for evaluating the ability of galectin-3 to identify patients with diastolic dysfunction grade III

\begin{tabular}{lllc}
\hline & $\begin{array}{c}\text { Adjusted odds } \\
\text { ratio }\end{array}$ & 95\% Cl & Adjusted $P$ \\
\hline Galectin-3 ( $\geq 17.0 \mathrm{ng} / \mathrm{mL})$ & 6.19 & $1.489-25.744$ & $0.012^{*}$ \\
NT-proBNP $(\geq 290.6 \mathrm{pg} / \mathrm{mL})$ & 2.667 & $0.601-11.830$ & 0.197 \\
Gender & 1.244 & $0.295-5.246$ & 0.767 \\
Creatinine & 1.33 & $0.367-4.825$ & 0.664 \\
LA $(>45 \mathrm{~mm})$ & 1.855 & $0.411-8.379$ & 0.422 \\
\hline
\end{tabular}

*Values were adjusted to creatinine, so no cut-off values were required. Abbreviation: $\mathrm{Cl}$, confidence interval; LA, left atrium; NT-proBNP, amino-terminal pro-brain natriuretic peptide.

6.19, 95\% Cl 1.489-25.744, $P=0.012$ ). The likelihood of developing this severe form of HFpEF was two-to-three times greater in patients with NT-proBNP levels $\geq 290.6 \mathrm{pg} / \mathrm{mL}(\mathrm{OR}=-2.667$,
95\% Cl 0.601-11.830, $P=0.197$; Table 4). An analysis of logtransformed NT-proBNP levels alone showed statistical insignificance in this scenario.

\section{DISCUSSION}

This study aimed to determine the relationship between Gal-3 levels and the echocardiographic indices corresponding to various stages of HFpEF (LVEF > 50\%). At the outset, Gal-3 proved effective in the diagnosis of patients suffering from a severe grade of diastolic dysfunction. The ability to diagnose patients with HFpEF exhibited some similarity with the reference biomarker, NTproBNP. Interestingly, increasing levels of Gal-3 also possibly reflected the progressive course of $\mathrm{HFpEF}$, as evident in the echocardiographic examination; indices measuring atrial and ventricular dimensions were noticeably altered from their normal 


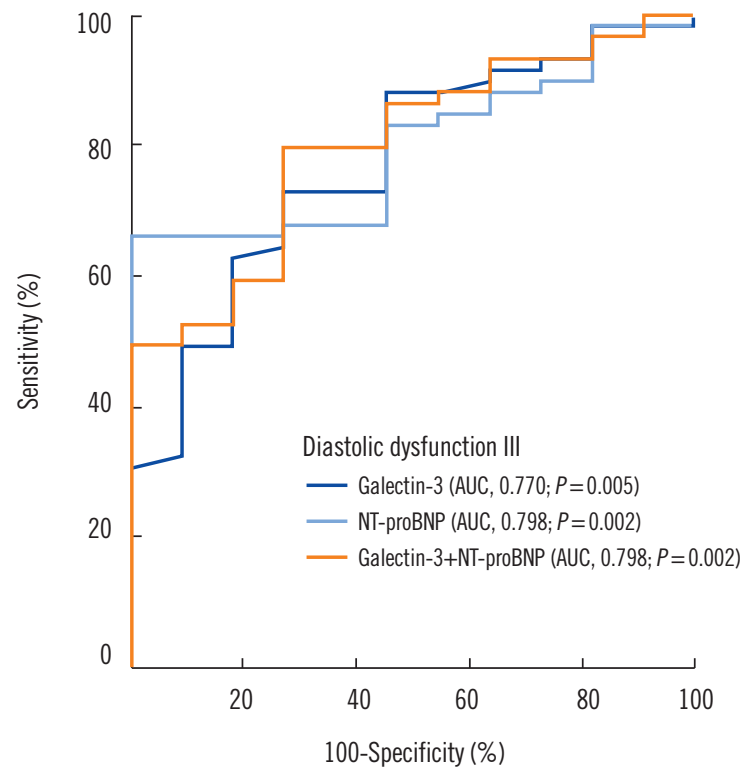

Fig. 2. Receiver-operating characteristic (ROC) curve revealing an effective discrimination of patients with diastolic dysfunction III. ROC = diastolic dysfunction grade 3 curve. AUC-Area under the Curve. Abbreviation: NT-proBNP, amino-terminal pro-brain natriuretic peptide.

range. Furthermore, the combination of Gal-3 and NT-proBNP values was equally effective in identifying patients with grade III diastolic dysfunction.

Several clinical studies have attempted to elucidate the role of Gal-3 in HFpEF patients [17]. These include the Coordinating study evaluating Outcomes of Advising and Counselling in Heart Failure ( $\mathrm{COACH})$ trial [34], the Controlled Rosuvastatin Multinational Trial in Heart Failure (CORONA) cohort [34], and studies by Carrasco-Sanchez et al [16], which described the strong prognostic value of Gal-3 in HFpEF patients [18]. However, the potential of Gal-3 to reflect the evolving stages of diastolic dysfunction remains poorly understood; our study has attempted to demonstrate this distinct correlation.

Gal-3 is essentially a product of active macrophages with binding sites on cardiac-resident fibroblasts, mechanistically influencing increased myocardial collagen expression, interstitial fibrosis, TGF- $\beta$ activation, and subsequent LV dysfunction [1921]. Its role in response to injury and inflammation in heart failure is further supplemented by a significant contribution to ventricular remodeling [12]. This study provides useful information corroborating the association between LV structure and function and Gal-3 level, essentially mirroring the hypothesized link between cardiac fibrosis, hypertrophy, and evolving heart failure. Higher LV filling pressures (E/Ea ratio) and the varying degrees of diastolic dysfunction ( $E_{a}$ velocity, early diastolic myocardial re- laxation velocity below the baseline as the annulus ascends away from the apex) are probable effects of cardiac stiffness mediated by Gal-3.

The identification of Gal-3 as a potential predictive biomarker of HFpEF was suggested by a sub-study of the Pro-BNP Investigation of Dyspnea in the Emergency Department (PRIDE) trial, in which echocardiographic indices reflecting diastolic dysfunction showed a strong correlation with measured serum levels [22]. Subsequently, Zile et al [18] demonstrated that Gal-3 levels were significantly elevated in an HFpEF patient cohort. Further studies by de Boer et al [17], involving cohorts enlisted in the $\mathrm{COACH}$ study, also garnered substantial interest by suggesting that patients with $\mathrm{HFpEF}$ had a much stronger correlation with Gal-3 than those with reduced EF. The contribution of arterial hypertension in the development of HFpEF is undisputed, and it has been suggested that this interaction could also be influenced by Gal-3. Large community-based cohorts in which Gal-3 levels were measured (Prevention of Renal and Vascular End-Stage Disease and Framingham Offspring-PREVEND) demonstrated a convincing relationship with blood pressure values, as well as long-term mortality [23]. We believe that the present study has also successfully validated this relationship.

Although tangible data from the Deventer-Alkmaar HF Project (DEAL-HF) and Aldosterone Receptor Blockade in Diastolic Heart Failure (ALDO-DHF) trials complement the view that Gal-3 level demonstrates a prognostic value regardless of heart failure severity [24, 25], early interpretation of these results has raised some criticism. For example, the association of Gal-3 level and risk of new-onset heart failure among patients included in the Framingham Heart study was statistically nonsignificant in patients suffering from chronic kidney disease [26]. Furthermore, a short summary of published trials (RELAX-Phosphodiesterase-5 Inhibition to Improve Clinical Status and Exercise Capacity in Heart Failure with Preserved Ejection Fraction Trial and ALDOHF) by deFilippi and Christenson [26, 27], has suggested that Gal-3 levels are not associated with significant cardiac structural or functional abnormalities, as determined by cardiovascular imaging, and are principally linked to renal function. Although results from new studies (REGAL-HF) are still awaited, there exists a growing body of evidence questioning this assertion. To better understand this dilemma, a retrospective study was initiated to examine the correlation between Gal-3 level and GFR. This study revealed a continual relationship after adjustment for age, LVEF, and NT-proBNP [28] and further implied that it is renal impairment (with signs of inflammation and fibrosis) that contributes to the prognostic properties of Gal-3 in HFpEF. Sup- 
plementary work by Gurel et al [29] suggested the potential use of Gal-3 in detecting HFpEF in patients undergoing hemodialysis. The present results demonstrate a significant relationship between Gal-3 levels and patient serum creatinine values across the entire HFpEF spectrum of patients. Additionally, the correlation between Gal-3 level and diastolic dysfunction has also been shown to evolve along the course of the HFpEF syndrome. It is well known that renal dysfunction is one of the most powerful predictors of prognosis in heart failure, suggesting the possible existence of an overlap between these syndromes, mediated at a pathophysiological level by Gal-3, which could explain this contradictory evidence [30].

Another point for debate is the common use of NT-proBNP, the standard biomarker for diagnosing different forms of heart failure. Our study showed no significant advantage for Gal-3 over NT-proBNP in diagnosing HFpEF, thus raising questions regarding the need for Gal-3 as an additional biomarker. As HFpEF and the various grades of diastolic dysfunction are dynamic and diagnosed based on echocardiographic measurements, it is pertinent to accurately ascertain the level of dysfunction in the patient so as to tailor pharmacological therapy. Additionally, early diagnosis plays a key role in syndrome management. Based on our results, it could be argued that the combined use of both these biomarkers would enable more effective diagnosis and categorization of heart failure stage. Furthermore, the effectiveness of Gal-3 as a serological biomarker is cemented by the hypothesis that the ongoing cardiac fibrotic process is irreversible; Gal-3 levels remain unchanged in the event of acute cardiac decompensation and are not influenced by medical treatment, as is the case for NT-proBNP [31].

In summary, Gal-3 is a promising novel biomarker with the potential to classify patients on the cusp of HFpEF syndrome. Its preferred use with other biomarkers, such as NT-proBNP, may reveal certain HFpEF states that are not evident in routine clinical examination. This would definitely be helpful to the segment of the patient population that cannot be diagnosed because of echocardiography limits. A recent clinical guidelines update issued by the American Heart Association/American College of Cardiology (AHA/ACC) emphasized the role of Gal-3 as a predictor of mortality and hospitalization in patients with heart failure, suggesting a class $\mathrm{Ilb}$ recommendation in this setting $[32,33]$. Although initial data from the ALDO-HF trials showed no significant interaction between spironolactone and Gal-3 levels and the TOPCAT (Treatment of Preserved Cardiac Function Heart Failure with Aldosterone Antagonist) trial revealed further uncertainties in this regard, the optimization of pharmacological therapies, which could be possibly influenced by Gal-3 measurements, needs to be further evaluated. Eagerly awaited results from the REGAL-HF trial will probably define these strategies in future.

This prospective study was inherently limited by its small size and the nature of the patient cohort. For example, the representative population was sampled from those presenting for routine echocardiography at an outpatient department in various stages of heart failure, with a bias skewed towards patients displaying at least some syndrome symptoms. Furthermore, the echocardiographic evaluation, although executed according to European Society of Cardiology (ESC) standards, was carried out by at least three different examiners with varying levels of expertise, thus offering a certain level of discrepancy in its interpretation. The use of a single time point reference for sampling Gal-3 levels limited the interpretation of these results to the six- and 12-month follow-up. It is therefore debatable if the progression of diastolic dysfunction, as measured by echocardiography, will correspond to future Gal-3 levels. Additionally, the number of patients with severe diastolic dysfunction was too low to include five or more variables in the multivariate model, which could hinder meaningful multivariate analysis. However, this has been added to our study to demonstrate the statistical consistency within our cohort, which could be reevaluated in larger studies in the future.

This study demonstrates that Gal-3 is effective in diagnosing patients suffering from a severe grade of diastolic dysfunction and that the simultaneous use of NT-proBNP could reflect the increased probability of disease in doubtful scenarios. Additionally, increasing levels of serum Gal-3 could also reflect the progressive course of $\mathrm{HFpEF}$, as classified by the echocardiographic grades of diastolic dysfunction. Thus, this biomarker may be helpful for patients in cases in which echocardiography interpretation is limited. As these results reflect a small patient pool, further studies examining the potential of these markers are definitely warranted.

\section{Authors' Disclosures of Potential Conflicts of Interest}

Thomas Bertsch performs reagent evaluation studies for Roche Diagnostics. All other authors declare that they have no competing interests.

\section{Acknowledgment}

This study was supported by the Deutsches Zentrum fur Herz- 
Kreislauf-Forschung-German Centre for Cardiovascular Research (DZHK).

\section{REFERENCES}

1. Go AS, Mozaffarian D, Roger VL, Benjamin EJ, Berry JD, Blaha MJ, et al. Heart disease and stroke statistics--2014 update: a report from the American Heart Association. Circulation 2014;129:e28-e292.

2. McMurray JJ, Adamopoulos S, Anker SD, Auricchio A, Böhm M, Dickstein $\mathrm{K}$, et al. ESC guidelines for the diagnosis and treatment of acute and chronic heart failure 2012: The Task Force for the Diagnosis and Treatment of Acute and Chronic Heart Failure 2012 of the European Society of Cardiology. Developed in collaboration with the Heart Failure Association (HFA) of the ESC. Eur Heart J 2012;33:1787-847.

3. Cheng JM, Akkerhuis KM, Battes LC, van Vark LC, Hillege HL, Paulus WJ, et al. Biomarkers of heart failure with normal ejection fraction: a systematic review. Eur J Heart Fail 2013;15:1350-62.

4. de Boer RA, Edelmann F, Cohen-Solal A, Mamas MA, Maisel A, Pieske B. Galectin-3 in heart failure with preserved ejection fraction. Eur J Heart Fail 2013;15:1095-101.

5. Tribouilloy C, Rusinaru D, Mahjoub H, Souliere V, Levy F, Peltier M, et al. Prognosis of heart failure with preserved ejection fraction: a 5 year prospective population-based study. Eur Heart J 2008;29:339-47.

6. González A, López B, Díez J. Fibrosis in hypertensive heart disease: role of the renin-angiotensin-aldosterone system. Med Clin North Am 2004; 88:83-97.

7. Mitchell JA, Ventura HO, Mehra MR. Early recognition and treatment of hypertensive heart disease. Curr Opin Cardiol 2005;20:282-9.

8. de Boer RA, Pinto YM, Van Veldhuisen DJ. The imbalance between oxygen demand and supply as a potential mechanism in the pathophysiology of heart failure: the role of microvascular growth and abnormalities. Microcirculation 2003;10:113-26.

9. Henderson NC and Sethi T. The regulation of inflammation by Galectin-3. Immunol Rev 2009;230:160-71.

10. Reifenberg K, Lehr HA, Torzewski M, Steige G, Wiese E, Küppr I, et al. Interferon- $\gamma$ induces chronic active myocarditis and cardiomyopathy in transgenic mice. Am J Pathol 2007;171:463-72.

11. Lok DJ, Van Der Meer P, de la Porte PW, Lipsic E, van Wijngaarden J, Hillege HL, et al. Prognostic value of galectin-3, a novel marker of fibrosis, in patients with chronic heart failure: data from the DEAL-HF study. Clin Res Cardiol 2010;99:323-8.

12. Shah RV, Chen-Tournoux AA, Picard MH, van Kimmenade RR, Januzzi JL. Galectin-3, cardiac structure and function, and long-term mortality in patients with acutely decompensated heart failure. Eur J Heart Fail 2010;12:826-32.

13. Nagueh SF, Smiseth, OA, Appleton CP, Byrd BF 3rd, Dokainish H, Edvardsen $\mathrm{T}$, et al. Recommendations for the evaluation of left ventricular diastolic function by echocardiography: an update from the American Society of Echocardiography and the European Association of Cardiovascular Imaging. J Am Soc Echocardiogr 2016;29:277-314.

14. Penicka M, Vanderheyden M, Bartunek J. Diagnosis of heart failure with preserved ejection fraction: role of clinical Doppler echocardiography. Heart 2014;100:68-76.

15. Gruson D, Mancini M, Ahn SA, Rousseau MF. Measurement of Galectin-3 with the ARCHITECT assay: Clinical validity and cost-effectiveness in patients with heart failure. Clin Biochem 2014;47:1006-9.

16. Carrasco-Sánchez FJ, Aramburu-Bodas O, Salamanca-Bautista P, Morales-Rull JL, Galisteo-Almeda L, Páez-Rubio MI, et al. Predictive value of serum galectin-3 levels in patients with acute heart failure with preserved ejection fraction. Int J Cardiol 2013;169:177-82.

17. de Boer RA, Lok DJ, Jaarsma T, van der Meer P, Voors AA, Hillege HL, et al. Predictive value of plasma galectin-3 levels in heart failure with reduced and preserved ejection fraction. Ann Med 2011;43:60-8.

18. Zile MR, DeSantis SM, Baicu CF, Prescott CB, Stroud RE, Thompson $\mathrm{SB}$, et al. Plasma galectin-3 levels in patients with structural and clinical manifestation of hypertensive heart disease: relationship to determination of matrix composition. Circulation 2010;122(S1):A12433.

19. Sharma UC, Pokharel S, van Brakel TJ, van Berlo JH, Cleutjens JP, Schroen B, et al. Galectin-3 marks activated macrophages in failure-prone hypertrophied hearts and contributes to cardiac dysfunction. Circulation 2004;110:3121-8.

20. Liu YH, d'Ambrosio M, Liu YD, Peng H, Rhaleb NE, Sharma U, et al. Nacetyl-seryl-aspartyl-lysyl-proline prevents cardiac remodeling and dysfunction induced by galectin-3, a mammalian adhesion/growth-regulatory lectin. Am J Physiol Heart Circ Physiol 2009;296:H404-12.

21. de Boer RA, Voors AA, Muntendam P, van Gilst WH, van Veldhuisen DJ. Galectin-3: a novel mediator of heart failure development and progression. Eur J Heart Fail 2009;11:811-7.

22. van Kimmenade RR, Januzzi JL Jr, Ellinor PT, Sharma UC, Bakker JA, Low AF, et al. Utility of amino-terminal pro-brain natriuretic peptide, galectin-3, and apelin for the evaluation of patients with acute heart failure. J Am Coll Cardiol 2006;48:1217-24.

23. Ho JE, Liu C, Lyass A, Courchesne P, Pencina MJ, Vasan RS, et al. Galectin-3, a marker of cardiac fibrosis, predicts incident heart failure in the community. J Am Coll Cardiol 2012;60:1249-56.

24. de Boer RA, van Veldhuisen DJ, Gansevoort RT, Muller Kobold AC, van Gilst WH, Hillege HL, et al. The fibrosis marker galectin-3 and outcome in the general population. J Intern Med 2012;272:55-64.

25. Edelmann F, Holzendorf V, Wachter R, Nolte K, Schmidt AG, KraigherKrainer $\mathrm{E}$, et al. Galectin-3 in patients with heart failure with preserved ejection fraction: results from the Aldo-DHF trial. Eur J Heart Fail 2015; 17:214-23.

26. deFilippi $\mathrm{CR}$ and Christenson RH. Evolving role of galectin-3 as a cardiac biomarker. Heart failure with preserved ejection fraction and renal function, important pieces of the puzzle. JACC Heart Fail 2015;3:253-6.

27. AbouEzzeddine OF, Haines P, Stevens S, Nativti-Nicolau J, Felker GM, Borlaug BA, et al. Galectin-3 in heart failure with preserved ejection fraction: a RELAX trial substudy (phosphodiesterase-5 inhibition to improve clinical status and exercise capacity in diastolic heart failure). JACC Heart Fail 2015;3:245-52.

28. Gopal DM, Kommineni M, Ayalon N, Koelbl C, Ayalon R, Biolo A, et al. Relationship of plasma galectin-3 to renal function in patients with heart failure: effects of clinical status, pathophysiology of heart failure, and presence or absence of heart failure. J Am Heart Assoc 2012;1:e000760.

29. Gurel OM, Yilmaz H, Celik TH, Cakmak M, Namuslu M, Bilgiç AM, et al. Galectin-3 as a new biomarker of diastolic dysfunction in hemodialysis patients. Herz 2015;40:788-94.

30. Smilde TD, Damman K, van der Harst P, Navis G, Westenbrink BD, Voors $A A$, et al. Differential associations between renal function and "modifiable" risk factors in patients with chronic heart failure. Clin Res Cardiol 2009;98:121-9.

31. Bošnjak I, Selthofer-Relatić K, Včev A. Prognostic value of galectin-3 in patients with heart failure. Dis Markers 2015;2015:690205.

32. Christenson RH, Duh SH, Wu AHB, Smith A, Abel G, deFilippi CR, et al. Multi-center determination of galectin-3 assay performance characteristics: anatomy of a novel assay for use in heart failure. Clin Biochem 2010;43:683-90.

33. Yancy CW, Jessup M, Bozkurt B, Butler J, Casey DE Jr, Drazner MH, et 
Ansari U, et al.

Gal-3 reflects LV diastolic dysfunction severity

al. 2013 ACCF/AHA guideline for the management of heart failure: a report of the American College of Cardiology Foundation/American Heart Association Task Force on Practice Guidelines. J Am Coll Cardiol 2013; 62:e147-239.
34. Van der Velde A, Gullestad L, Ueland T, Aukrust P, Guo Y, Adourian A, et al. 2013. Prognostic Value of Changes in Galectin-3 levels over time in Patients with Heart failure. Clinical Perspective Data from CORONA and CÓACH. Circulation: Heart Failure. 2013;6:219-26. 